\title{
Spinning Relativistic Particles in External Fields
}

\author{
I.B. Khriplovich 1 \\ Budker Institute of Nuclear Physics, 630090 Novosibirsk, Russia, \\ and Novosibirsk University
}

\begin{abstract}
The motion of spinning relativistic particles in external electromagnetic and gravitational fields is considered. A simple derivation of the spin interaction with gravitational field is presented. The self-consistent description of the spin corrections to the equations of motion is built with the noncovariant description of spin and with the usual, "naïve" definition of the coordinate of a relativistic particle.
\end{abstract}

\section{Introduction}

The pioneering paper [1] by Myron Mathisson on the relativistic equations of motion for spinning particle in a gravitational field was published in Acta Physica Polonica. I truly appreciate the invitation to submit a review on the subject to this journal.

The general problem of the motion of a relativistic particle with internal angular momentum (spin) in external electromagnetic and gravitational fields consists of two parts: the description of the spin precession and accounting for the spin influence on the trajectory of particle motion.

As to the first part of the problem, that of the spin evolution by itself, it is essentially settled, starting with the paper by Mathisson [1] (see also [2]). A relatively simple derivation of the corresponding relativistic equations, both in electrodynamics and gravity, is presented below, together with the detailed discussion of the limits of applicability for these equations.

The situation is different with the second part of the problem, i.e., how the interaction of spin with an external field influences the trajectory of a particle. On the one hand, there is an old prejudice according to which for elementary particles this influence is unobservable by virtue of the uncertainty relation. On the other hand, there are serious disagreements on the exact form of the effect.

The discussed effect in electromagnetic field is of real physical interest, being related to the problem of separating different polarizations of relativistic particles in accelerators. However, as mentioned, it is not as obvious whether one can observe in practice the discussed spin corrections to the equations of motion of elementary particles, for instance, electron or proton. According to the well-known observation by Bohr (quoted in [3]), an additional Lorentz force due to the finite size of the wave packet of a charged particle and to the uncertainty relation, is on the same order of magnitude as the corresponding component of the Stern-Gerlach force. However, this argument by itself does not exclude in principle the possibility to observe a regular Stern-Gerlach effect, even a small one, in the presence of a comparable background due to the uncertainty relation. It is sufficient to recall quite

\footnotetext{
${ }^{1}$ khriplovich@inp.nsk.su
} 
common situation when the accuracy with which the energy of an unstable level is known, is much better than the width of this level.

Besides, not only spin-dependent correlations certainly exist in differential cross sections of scattering processes, but they are effectively used to separate particles of different polarizations. That is why it was proposed long ago to separate charged particles of different polarizations in a storage ring through the spin interaction with external fields [4]. Though this proposal is discussed rather actively (see, for instance, review [5]), it is not clear up to now whether it is feasible technically.

On the other hand, certainly there are macroscopic objects for which internal rotation influences their trajectories. We mean the motion of Kerr black holes in external gravitational fields. This problem is of importance in particular for the calculation of the gravitational radiation of binary stars. In this connection it was considered in [6-9]. However, the equations of motion taking account of the spin influence to lowest nonvanishing order in $c^{-2}$, used in these papers, lead to results which differ from the well-known gravitational spin-orbit interaction even in the simpler case of an external field. As will be demonstrated below, the disagreement originates from different definitions of the center-of-mass coordinate. In the analysis of this disagreement and in the solution of the problem we follow [10, 11].

\section{Equations of Motion of Spin in Electromagnetic Field}

The equations of motion for spin of a relativistic particle in electromagnetic field are well known. However, since the general problem of the spin interaction with external gravitational field reduces to the analogous problem for the case of an external electromagnetic field, it is pertinent to start the detailed analysis of the subject in this way. Besides, the subtleties with the definition of the center-of-mass coordinate can be essentially elucidated in this problem, which is somewhat more simple than the gravitational one.

\subsection{Covariant Equation of Motion of Spin}

Let us consider at first the spin precession for a nonrelativistic charged particle. The equation that describes this precession is well known:

$$
\dot{\mathbf{s}}=\frac{e g}{2 m}[\mathbf{s} \times \mathbf{B}] .
$$

Here $\mathbf{B}$ is an external magnetic field, $e$ and $m$ are the charge and mass of the particle, $g$ is its gyromagnetic ratio (for electron $g \approx 2$ ). In other words, the spin precesses around the direction of magnetic field with the frequency $-(\mathrm{eg} / 2 \mathrm{~m}) \mathbf{B}$. In the same nonrelativistic limit the velocity precesses around the direction of $\mathbf{B}$ with the frequency $-(e / m) \mathbf{B}$ :

$$
\dot{\mathbf{v}}=\frac{e}{m}[\mathbf{v} \times \mathbf{B}]
$$

Thus, for $g=2$ spin and velocity precess with the same frequency, so that the angle between them is conserved. 
Let us note that both equations, (1) and (2), hold as Heisenberg equations of motion in an external field for the spin and velocity operators, s and $\mathbf{v}$. On the other hand, being averaged over properly localized wave packets, these equations go over into the (semi)classical equations of motion for spin and velocity. This refers also to the relativistic generalizations of equation (1), discussed in this section below.

We will consider at first the covariant semiclassical formalism using the four-dimensional vector of spin $S_{\mu}$. This 4 -vector is defined as follows. In the particle rest frame $S_{\mu}$ has no time component and reduces to the common three-dimensional vector of spin s, i.e., in this frame $S_{\mu}=(0, \mathbf{s})$. In the reference frame where the particle moves with velocity $\mathbf{v}$, the vector $S_{\mu}$ is constructed from $(0, \mathbf{s})$ by means of the Lorentz transformation, so that here

$$
S_{0}=\gamma \mathbf{v s}, \quad \mathbf{S}=\mathbf{s}+\frac{\gamma^{2} \mathbf{v}(\mathbf{v s})}{\gamma+1} .
$$

Then, just by definition of $S_{\mu}$, the following identities take place:

$$
S_{\mu} S_{\mu}=-\mathbf{s}^{2}(=\mathrm{const}), \quad S_{\mu} u_{\mu}=0
$$

as usual, here $u_{\mu}$ is the four-velocity. It should be emphasized that it is just the velocity, which is a parameter of the Lorentz group, that enters these relations (but not the canonical momentum, which is not even a gauge-invariant vector).

The right-hand side of the equation for $d S_{\mu} / d \tau$ (here and below $\tau$ is the proper time) should be linear and homogeneous both in the electromagnetic field strength $F_{\mu \nu}$, and in the same four-vector $S_{\mu}$, and may depend also on $u_{\mu}$. By virtue of the first identity (4), the right-hand side should be four-dimensionally orthogonal to $S_{\mu}$. Therefore, the general structure of the equation we are looking for is

$$
\frac{d S_{\mu}}{d \tau}=\alpha F_{\mu \nu} S_{\nu}+\beta u_{\mu} F_{\nu \lambda} u_{\nu} S_{\lambda}
$$

Comparing the nonrelativistic limit of this equation with (1), we find

$$
\alpha=\frac{e g}{2 m} .
$$

Now we take into account the second identity (44), which after differentiation in $\tau$ gives

$$
u_{\mu} \frac{d S_{\mu}}{d \tau}=-S_{\mu} \frac{d u_{\mu}}{d \tau}
$$

and recall the classical equation of motion for a charge:

$$
m \frac{d u_{\mu}}{d \tau}=e F_{\mu \nu} u_{\nu}
$$

Then, multiplying equation (5) by $u_{\mu}$, we obtain

$$
\beta=-\frac{e}{2 m}(g-2) .
$$


Thus, the covariant equation of motion for spin is [12-14]

$$
\frac{d S_{\mu}}{d \tau}=\frac{e g}{2 m} F_{\mu \nu} S_{\nu}-\frac{e}{2 m}(g-2) u_{\mu} F_{\nu \lambda} u_{\nu} S_{\lambda} .
$$

Let us discuss the limits of applicability for this equation.

Of course, typical distances at which the trajectory changes (for instance, the Larmor radius in a magnetic field) should be large as compared to the de Broglie wave length $\hbar / p$ of the elementary particle. Then, the external field itself should not change essentially at the distances on the order of both the de Broglie wave length $\hbar / p$ and the Compton wave length $\hbar /(m c)$ of the particle. In particular, if the last condition does not hold, the scatter of velocities in the rest frame is not small as compared to $c$, and one cannot use in this frame the nonrelativistic formulae.

Besides, if the external field changes rapidly, the motion of spin will be influenced by interaction of higher electromagnetic multipoles of the particle with field gradients. For a particle of spin 1/2 higher multipoles are absent, and the gradient-dependent effects are due to finite form factors of the particle. These effects start here at least in second order in field gradients and usually are negligible.

At last, in equation (7) we confine to effects of first order in the external field. This approximation relies in fact on the implicit assumption that the first-order interaction with the external field is less than the excitation energy of the spinning system. Usually this assumption is true and the first-order equation (17) is valid. Still, one can easily point out situations when this is not the case. To be definite, let us consider the hydrogen-like ion ${ }^{3} \mathrm{He}^{+}$ in the ground $s$-state with the total spin $F=1$. It can be easily demonstrated that an already quite moderate external magnetic field is sufficient to break the hyperfine interaction between the electron and nuclear magnetic moments (a sort of Paschen-Back effect). Then, instead of a precession of the total spin $\mathbf{F}$ of the ion, which should be described by equations (11) or (7) with a corresponding ion $g$-factor, we will have a separate precession of the decoupled electron and nuclear spins.

Let us go back now to equation (7). We note that for $g=2$ and in the absence of electric field, its zeroth component reduces to

$$
\frac{d S_{0}}{d \tau}=0
$$

Taking into account definition (3) for $S_{0}$ and the fact that in a magnetic field a particle energy remains constant, we find immediately that the projection of spin s onto velocity, so-called helicity, is conserved.

\subsection{Noncovariant Equation of Motion for Spin of Relativistic Particle. Thomas Precession}

We will obtain now the relativistic equation for the three-dimensional vector of spin $\mathbf{s}$, that directly describes the internal angular momentum of a particle in its "momentary" rest frame. This equation can be derived from (7) using relations (3), together with the equations 
of motion for a charge in external field. It will require, however, quite tedious calculations. Therefore, we choose another way, somewhat more simple and much more instructive.

First, we transform equation (11) from the comoving inertial frame, where the particle is at rest, into the laboratory one. The magnetic field $\mathbf{B}^{\prime}$ in the rest frame is expressed via the electric and magnetic fields $\mathbf{E}$ and $\mathbf{B}$ given in the laboratory frame, as follows:

$$
\mathbf{B}^{\prime}=\gamma \mathbf{B}-\frac{\gamma^{2}}{\gamma+1} \mathbf{v}(\mathbf{v B})-\gamma \mathbf{v} \times \mathbf{E} .
$$

This expression can be easily checked by comparing it component by component with the transformation of magnetic field for two cases: when this field is parallel to the velocity and orthogonal to it, respectively. Then one should take into account that the frequency in the laboratory time $t$ is $\gamma$ times smaller than the frequency in the laboratory time $\tau$ (indeed, $\left.d / d t=d \tau / d t \cdot d / d \tau=\gamma^{-1} d / d \tau\right)$. Found in this way contribution to the precession frequency is

$$
\boldsymbol{\omega}_{g}=-\frac{e g}{2 m}\left[\mathbf{B}-\frac{\gamma}{\gamma+1} \mathbf{v}(\mathbf{v B})-\mathbf{v} \times \mathbf{E}\right] .
$$

However it is clear from equation (17) that spin precesses even if $g=0$. To elucidate the origin of this effect, the so-called Thomas precession [15], we consider two successive Lorentz transformations: at first from the laboratory frame $S$ into the frame $S^{\prime}$ that moves with the velocity v with respect to $S$, and then from $S^{\prime}$ into the frame $S^{\prime \prime}$ that moves with respect to $S^{\prime}$ with the infinitesimal velocity $d \mathbf{v}$. Let us recall in this connection the following fact related to usual three-dimensional rotations: the result of two successive rotations with respect to noncollinear axes $\mathbf{n}_{1}$ and $\mathbf{n}_{2}$ contains in particular a rotation around the axis directed along their vector product $\mathbf{n}_{1} \times \mathbf{n}_{2}$. Now it is only natural to assume that the result of the above successive Lorentz transformations will contain in particular a usual rotation around the axis directed along $d \mathbf{v} \times \mathbf{v}$. In result, spin in the rest frame will rotate in the opposite direction by an angle which we denote by $\kappa[d \mathbf{v} \times \mathbf{v}]$. Here $\kappa$ is some numerical factor to be determined below. It depends generally speaking on the particle energy.

This is in fact the Thomas precession. Its frequency in the proper time $\tau$ is

$$
\boldsymbol{\omega}_{T}^{\prime}=\kappa[d \mathbf{v} / d \tau \times \mathbf{v}]=\kappa \frac{e}{m}\left[\mathbf{E}^{\prime} \times \mathbf{v}\right] .
$$

Now we transform the electric field $\mathbf{E}^{\prime}$ from the proper frame into the laboratory one, as it was done above for the magnetic field $\mathbf{B}^{\prime}$, and go over also from the proper time $\tau$ to $t$. In result, the frequency of the Thomas precession in the laboratory frame is

$$
\begin{aligned}
\boldsymbol{\omega}_{T} & =\kappa \frac{e}{m}\left[\left(\mathbf{E}-\frac{\gamma}{\gamma+1} \mathbf{v}(\mathbf{v E})+\mathbf{v} \times \mathbf{B}\right) \times \mathbf{v}\right] \\
& =-\kappa \frac{e}{m}\left[\mathbf{v} \times \mathbf{E}-v^{2} \mathbf{B}+\mathbf{v}(\mathbf{v B})\right] .
\end{aligned}
$$

To find the coefficient $\kappa$, we recall that in a magnetic field, for $g=2$ the projection of spin onto the velocity is conserved. In other words, in this case the total frequency of the spin 
precession $\boldsymbol{\omega}=\boldsymbol{\omega}_{g}+\boldsymbol{\omega}_{T}$ coincides with the frequency of the velocity precession which is well known to be

$$
\boldsymbol{\omega}_{v}=-\frac{e}{m \gamma} \mathbf{B}
$$

From this we find easily that $\kappa=\gamma /(\gamma+1)$. Correspondingly, the relativistic equation of motion for the three-dimensional vector of spin $\mathbf{s}$ in external electromagnetic field is

$$
\begin{gathered}
\frac{d \mathbf{s}}{d t}=\left(\boldsymbol{\omega}_{g}+\boldsymbol{\omega}_{T}\right) \times \mathbf{s}=\frac{e}{2 m}\left\{\left(g-2+\frac{2}{\gamma}\right)[\mathbf{s} \times \mathbf{B}]\right. \\
\left.-(g-2) \frac{\gamma}{\gamma+1}[\mathbf{s} \times \mathbf{v}](\mathbf{v B})-\left(g-\frac{2 \gamma}{\gamma+1}\right)[\mathbf{s} \times[\mathbf{v} \times \mathbf{E}]]\right\} .
\end{gathered}
$$

\subsection{Relativistic Spin Hamiltonian}

The relativistic Hamiltonian for the interaction of the three-dimensional vector of spin with external electromagnetic field is written in the usual form:

$$
H=\omega \mathbf{s} .
$$

Not only does it generate via the standard relation

$$
\frac{d \mathbf{s}}{d t}=\frac{i}{\hbar}[H, \mathbf{s}]
$$

equation (8). For instance, it is easy to obtain with this Hamiltonian equations of motion of the quadrupole moment of a relativistic particle in electric and magnetic fields, neglecting the field gradients. In the particle rest frame, the operator of its quadrupole moment is

$$
q_{m n}=\frac{3 q}{2 s(2 s-1)}\left[s_{m} s_{n}+s_{n} s_{m}-\frac{2}{3} s(s+1) \delta_{m n}\right]
$$

here the structure in square brackets guarantees the symmetry and vanishing trace of this operator, $q_{m n}=q_{n m}, q_{m m}=0$; the overall factor at the square brackets corresponds to the normalization condition $q_{z z}=q$ for $s_{z}=s$. To calculate the commutator in the corresponding equation

$$
\frac{d q_{m n}}{d t}=\frac{i}{\hbar}\left[\omega_{k} s_{k}, q_{m n}\right]
$$

is an elementary problem.

Moreover, Hamiltonian (9) is effective for the solution of various other physical problems, from the single-photon radiative transition between atomic $s$-levels to the low-energy theorems for Compton scattering. So, the validity of this Hamiltonian is beyond any doubts.

In particular, interaction (9) can be effectively used to derive the additional spin-dependent force acting upon a charged particle in an external electromagnetic field [16]. However, since $\boldsymbol{\omega}$ depends directly on the particle velocity $\mathbf{v}$ (but not on its momentum $\mathbf{p}$ ), it is somewhat more convenient to employ here the Lagrangian (but not Hamiltonian) formalism with

$$
L=-\omega \mathrm{s} .
$$

We will come back to the spin-dependent forces later. 


\section{Spin Precession in Gravitational Field}

In this section we present a simple and general derivation of the equations of the spin precession in a gravitational field (restricting to first order in spin), based on a remarkable analogy between gravitational and electromagnetic fields. Due to this correspondence, the formulae of the previous section are naturally adapted for the case of an external gravitational field. In this way we easily reproduce and generalize known results for gravitational spin effects.

\subsection{General Relations}

It follows from the angular momentum conservation in flat space-time taken together with the equivalence principle that the 4-vector of spin $S^{\mu}$ is parallel transported along the particle world-line. The parallel transport of a vector along a geodesic $x^{\mu}(\tau)$ means that its covariant derivative vanishes:

$$
\frac{D S^{\mu}}{D \tau}=0
$$

We will use the tetrad formalism natural for the description of spin. The tetrad components of spin

$$
S^{a}=S^{\mu} e_{\mu}^{a}
$$

(by the first letters of the Latin alphabet, $a, b, c, d$, we label here and below four-dimensional tetrad indices) behave as vectors under Lorentz transformations of the locally inertial frame. However, they do not change under generally covariant transformations $x^{\mu}=f^{\mu}\left(x^{\prime}\right)$. In other words, the four components $S^{a}$ are world scalars. Therefore, in virtue of relation (13), the equations for them appear as follows:

$$
\frac{d S^{a}}{d \tau}=\frac{D S^{a}}{D \tau}=S^{\mu} e_{\mu ; \nu}^{a} u^{\nu}=\eta^{a b} \gamma_{b c d} u^{d} S^{c} .
$$

The covariant derivative of a tetrad is by definition

$$
e_{\mu ; \nu}^{a}=\partial_{\nu} e_{\mu}^{a}-\Gamma_{\mu \nu}^{\kappa} e_{\kappa}^{a}
$$

and the quantity

$$
\gamma_{a b c}=e_{a \mu ; \nu} e_{b}^{\mu} e_{c}^{\nu}
$$

is the Ricci rotation coefficient. By means of covariant differentiation of the identity $e_{a \mu} e_{b}^{\mu}=$ $\eta_{a b}$, one can easily demonstrate that these coefficients are antisymmetric in the first pair of indices:

$$
\gamma_{a b c}=-\gamma_{b a c}
$$

Of course, the equations for the tetrad components of a 4-velocity look exactly in the same way as those for spin:

$$
\frac{d u^{a}}{d \tau}=\eta^{a b} \gamma_{b c d} u^{d} u^{c}
$$

The meaning of equations (14) and (17) is clear: the tetrad components of both vectors vary in the same way since their variation is due only to the rotation of the local Lorentz frame. 
There is a remarkable similarity between the discussed problem and the special case of $g=2$ in electrodynamics. According to equations (77) and (6), the four-dimensional spin and four-dimensional velocity of a charged particle with the gyromagnetic ratio $g=2$ precess with the same angular velocity:

$$
\frac{d S_{a}}{d \tau}=\frac{e}{m} F_{a b} S^{b}, \quad \frac{d u_{a}}{d \tau}=\frac{e}{m} F_{a b} u^{b} .
$$

In other words, the obvious correspondence takes place:

$$
\frac{e}{m} F_{a b} \longleftrightarrow \gamma_{a b c} u^{c} .
$$

It allows us to derive the precession frequency $\boldsymbol{\omega}$ of a three-dimensional vector of spin $\mathbf{s}$ in an external gravitational field from expression (8) by means of the simple substitution

$$
\frac{e}{m} B_{i} \longrightarrow-\frac{1}{2} \epsilon_{i k l} \gamma_{k l c} u^{c} ; \quad \frac{e}{m} E_{i} \longrightarrow \gamma_{0 i c} u^{c}
$$

(here again, it is just the velocity, a parameter of the local Lorentz group, that enters these relations, but not the canonical momentum). Thus, the precession frequency is

$$
\omega_{i}=\epsilon_{i k l}\left(\frac{1}{2} \gamma_{k l c}+\frac{u^{k}}{u^{0}+1} \gamma_{0 l c}\right) \frac{u^{c}}{u_{w}^{0}} .
$$

The factor $1 / u_{w}^{0}$ in expression (20) is due to the transition in the left-hand side of equation (14) to differentiating over the world time $t$ :

$$
\frac{d}{d \tau}=\frac{d t}{d \tau} \frac{d}{d t}=u_{w}^{0} \frac{d}{d t} .
$$

We supply here $u_{w}^{0}$ with the subscript $w$ to indicate that this is the world, but not the tetrad, component of 4 -velocity. All other indices in (20) are tetrad ones, $c=0,1,2,3, \quad i, k, l=$ $1,2,3$.

Naturally, the relativistic Hamiltonian for the interaction of the three-dimensional vector of spin with external gravitational field is exactly of the same form (9), as that in the electromagnetic case. Here, of course, the precession frequency $\boldsymbol{\omega}$ is given by formula (20).

As to the limits of applicability of the presented equations, which describe the spin precession in an external gravitational field, they are quite analogous to those pointed out in subsection 2.1 for the case of an electromagnetic field.

However, in some respect the spin interaction with a gravitational field differs essentially from that with an electromagnetic field. In the case of an electromagnetic field, the interaction depends, generally speaking, on a free phenomenological parameter, $g$-factor. Moreover, if one allows for the violation of invariance both under the reflection of space coordinates and under time reversal, one more parameter arises in the case of electromagnetic interaction, the value of the electric dipole moment of the particle. The point is that both magnetic and electric dipole moments interact with the electromagnetic field strength, so that this interaction is 
gauge-invariant for any value of these moments. Only the spin-independent interaction with the electromagnetic vector potential is fixed by the charge conservation and gauge invariance. On the contrary, the Ricci rotation coefficients $\gamma_{a b c}$ entering the gravitational first-order spin interaction (14), as distinct from the Riemann tensor, are noncovariant. Therefore, the discussed interaction of spin with gravitational field is fixed in unique way by the law of angular momentum conservation in flat space-time taken together with the equivalence principle, and thus it contains no free parameters [17].

On the other hand, it is no surprise that the precession frequency $\boldsymbol{\omega}$ depends not on the Riemann tensor, but on the rotation coefficients. Of course, this frequency should not be a tensor: it is sufficient to recall that a spin, which is at rest in an inertial reference frame, precesses in a rotating one.

\subsection{Spin-Orbit Interaction. Weak Field}

One can check easily that in the weak-field approximation where

$$
g_{\mu \nu}=\eta_{\mu \nu}+h_{\mu \nu}, \quad\left|h_{\mu \nu}\right| \ll 1,
$$

there is no difference between the tetrad and world indices in $e_{a \mu}$, and the tetrad appears as follows:

$$
e_{\mu \nu}=\eta_{\mu \nu}+\tilde{e}_{\mu \nu}, \quad\left|\tilde{e}_{\mu \nu}\right| \ll 1 .
$$

Relation between the tetrads and metric

$$
e_{a \mu} e_{b \nu} \eta^{a b}=g_{\mu \nu}
$$

in the weak-field approximation reduces to

$$
\tilde{e}_{\mu \nu}+\tilde{e}_{\nu \mu}=h_{\mu \nu}
$$

Under the demand that tetrads are expressed via metric only, one arrives at the so-called symmetric gauge for the tetrads where

$$
\tilde{e}_{\mu \nu}=\frac{1}{2} h_{\mu \nu}
$$

Then in the weak-field approximation the Ricci coefficients are:

$$
\gamma_{a b c}=\frac{1}{2}\left(h_{b c, a}-h_{a c, b}\right) .
$$

Now, with relations (20) and (21) one can solve, for instance, in an elementary way the problem of the gravitational spin-orbit interaction for arbitrary particle velocities. In the centrally symmetric field created by a mass $M$, the metric is

$$
h_{00}=-\frac{r_{g}}{r}=-\frac{2 k M}{r}, \quad h_{m n}=-\frac{r_{g}}{r} \delta_{m n}=-\frac{2 k M}{r} \delta_{m n} .
$$


Here the nonvanishing Ricci coefficients are

$$
\gamma_{i j k}=\frac{k M}{r^{3}}\left(\delta_{j k} r_{i}-\delta_{i k} r_{j}\right), \quad \gamma_{0 i 0}=-\frac{k M}{r^{3}} r_{i} .
$$

Plugging these expressions into formula (20) yields the following result for the precession frequency:

$$
\boldsymbol{\omega}_{l s}=\frac{2 \gamma+1}{\gamma+1} \frac{k M}{r^{3}} \mathbf{v} \times \mathbf{r} .
$$

The combination of a high velocity for a spinning particle with a weak gravitational field refers obviously to a scattering problem. Another possible application is to a spinning particle bound by other forces, for instance, by electromagnetic ones, when we are looking for the correction to the precession frequency due to the gravitational interaction.

In the limit of low velocities, $\gamma \rightarrow 1$, formula (24) goes over into the classical result [18]

$$
\boldsymbol{\omega}_{l s}=\frac{3}{2} \frac{k M}{r^{3}} \mathbf{v} \times \mathbf{r},
$$

with the gravitational spin-orbit potential 2

$$
U_{l s}(\mathbf{r})=\frac{3}{2} \frac{k M}{r^{3}}[\mathbf{v} \times \mathbf{r}] \mathbf{s} .
$$

\subsection{Spin-Orbit Interaction. Schwarzschild Field}

We consider now the spin precession in the Schwarzschild field beyond the weak-field approximation (though neglecting the spin influence on the trajectory). The 3-dimensional components of the Schwarzschild metric can be conveniently written as

$$
g_{m n}=-\left(\delta_{m n}-\frac{r_{m} r_{n}}{r^{2}}\right)-\frac{r_{m} r_{n}}{r^{2}} \frac{1}{1-r_{g} / r}=-\delta_{m n}^{\perp}-n_{m} n_{n} \frac{1}{1-r_{g} / r} .
$$

Nonvanishing tetrads are chosen as follows:

$$
e_{0}^{(0)}=\sqrt{1-r_{g} / r} ; \quad e_{m}^{(k)}=\delta_{k m}^{\perp}+n_{k} n_{m} \frac{1}{\sqrt{1-r_{g} / r}} ;
$$

in this subsection the tetrad indices are singled out by brackets. Now the nonvanishing Ricci coefficients (here their last indices are world ones) are

$$
\gamma_{(0)(i) 0}=-\frac{k M}{r^{3}} r_{i} ; \quad \gamma_{(i)(j) k}=\frac{1-\sqrt{1-r_{g} / r}}{r^{2}}\left(\delta_{j k} r_{i}-\delta_{i k} r_{j}\right) .
$$

At last, the precession frequency in this case is

$$
\boldsymbol{\omega}=-\mathbf{L} \frac{r_{g}}{2 m r^{3}}\left\{\frac{2}{u^{0}+u^{0} \sqrt{1-r_{g} / r}}+\frac{1}{1+u^{0} \sqrt{1-r_{g} / r}}\right\} .
$$

\footnotetext{
${ }^{2}$ It is curious that this result by Fokker for the gravitational spin-orbit interaction preceded by 5 years the corresponding one by Thomas for the electromagnetic case.
} 
Here $m$ and $\mathbf{L}$ are the particle mass and orbital angular momentum, respectively;

$$
u^{0}=\frac{d t}{d \tau}=\left\{1-r_{g} / r-(\mathbf{n v})^{2}\left(1-r_{g} / r\right)^{-1}-\left(\mathbf{v}^{\perp}\right)^{2}\right\}^{-1 / 2} .
$$

Rather cumbersome general expression (30) simplifies for a circular orbit. Here

$$
u^{0}=\left(1-\frac{3 k M}{r}\right)^{-1 / 2} ; \quad L=m r\left(\frac{k M}{r}\right)^{1 / 2}\left(1-\frac{3 k M}{r}\right)^{-1 / 2}
$$

so that

$$
\omega=\frac{(k M)^{1 / 2}}{r^{3 / 2}}\left[1-\left(1-\frac{3 k M}{r}\right)^{1 / 2}\right] .
$$

The general case of spin precession in the Schwarzschild field was considered in [19]. Our expression (31) agrees with the corresponding result of [19] (therein the precession is considered with respect to the proper time $\tau$, but not with respect to $t$ ).

\section{Covariant and Noncovariant Description of Spin-Dependent Forces}

\subsection{Problem with Covariant Formalism. Simple Example}

The difficulty with the covariant description of spin-dependent forces arises already for the electromagnetic interaction. To see what we are talking about, let us come back to Lagrangian (12). In the $c^{-2}$ approximation it generates the spin-dependent force

$$
f_{m}=\frac{e g}{2 m} \mathbf{s B},_{m}+\frac{e(g-1)}{2 m}\left(\frac{d}{d t}[\mathbf{E} \times \mathbf{s}]_{, m}-\mathbf{s}\left[\mathbf{v} \times \mathbf{E},_{m}\right]\right),
$$

acting on the particle (here and below in this subsection a subscript with a comma denotes a partial derivative).

Let us try to construct a covariant expression for the spin-dependent force acting on the particle, which would reproduce in the same $c^{-2}$ approximation force (32). Such covariant correction $f^{\mu}$ to the Lorentz force $e F^{\mu \nu} u_{\nu}$ should be linear in the tensor of spin $S_{\mu \nu}$ and in the gradient of the tensor of electromagnetic field $F_{\mu \nu, \lambda}$, it may depend also on the 4-velocity $u^{\mu}$. Since $u^{\mu} u_{\mu}=1$, this correction must satisfy the condition $u_{\mu} f^{\mu}=0$. From the mentioned tensors one can construct only two independent structures meeting the last condition. The first one,

$$
\eta^{\mu \kappa} F_{\nu \lambda, \kappa} S^{\nu \lambda}-F_{\lambda \nu, \kappa} u^{\kappa} S^{\lambda \nu} u^{\mu},
$$

reduces in the $c^{-2}$ approximation to

$$
2 \mathbf{s}\left(\mathbf{B},_{m}-\left[\mathbf{v} \times \mathbf{E},_{m}\right]\right),
$$


and the second one,

$$
u^{\lambda} F_{\lambda \nu, \kappa} u^{\kappa} S^{\nu \mu}
$$

reduces to

$$
\frac{d}{d t}[\mathbf{s} \times \mathbf{E}]_{, m}
$$

Let us note that possible structures with the contraction $F_{\nu \kappa, \lambda} S^{\kappa \lambda}$ reduce to (333) and (35), due to the Maxwell equations and the antisymmetry of $S_{\kappa \lambda}$.

Certainly, no linear combination of (34) and (36) can reproduce the correct expression (32) for the spin-dependent force.

But why is it that the correct (in the $c^{-2}$ approximation) formula (32) cannot be obtained from a covariant expression for the force? Obviously, one can easily reproduce by a linear combination of (34) and (36) those terms in (32) which are proportional to $g$. In other words, there is no problem to present in a covariant form the terms which describe, so to say, direct interaction of a magnetic moment with external fields. It is the terms in (32) independent of $g$ and corresponding to the Thomas precession, which cannot be written covariantly.

Of course, the noncovariance of equations by itself does not mean that physical observables have wrong transformation properties. It is sufficient to recall in this connection electrodynamics in the Coulomb gauge.

\subsection{What Is the Coordinate of Spinning Particle?}

The covariant formalism can be reconciled with the correct results if the coordinate $\mathbf{x}$ entering the covariant equation is related to the usual one $\mathbf{r}$ in the $c^{-2}$ approximation as follows:

$$
\mathbf{x}=\mathbf{r}+\frac{1}{2 m} \mathbf{s} \times \mathbf{v}
$$

The generalization of this substitution to the case of arbitrary velocities is [5]

$$
\mathbf{x}=\mathbf{r}+\frac{\gamma}{m(\gamma+1)} \mathbf{s} \times \mathbf{v}, \quad \gamma=\frac{1}{\sqrt{1-v^{2}}}
$$

Obviously, after this velocity-dependent substitution, the Lagrangian depends explicitly on the acceleration which in general results in spurious, nonphysical solutions.

Since relations (37), (38) are valid for a free spinning particle as well, their origin can be elucidated with a simple example of a free particle of spin $1 / 2$. Here, instead of the Dirac representation with the Hamiltonian of the standard form

$$
H=\alpha \mathbf{p}+\beta m,
$$

it is convenient to use the Foldy-Wouthuysen (FW) representation. In it the Hamiltonian is

$$
H_{F W}=\beta \varepsilon_{\mathbf{p}}, \quad \varepsilon_{\mathbf{p}}=\sqrt{\mathbf{p}^{2}+m^{2}},
$$


and the 4-component wave functions $\psi_{ \pm}$of the states of positive and negative energies reduce in fact to the 2-component spinors $\phi_{ \pm}$:

$$
\psi_{+}=\left(\begin{array}{c}
\phi_{+} \\
0
\end{array}\right), \quad \psi_{-}=\left(\begin{array}{c}
0 \\
\phi_{-}
\end{array}\right) .
$$

Obviously, in this representation the operator of coordinate $\hat{\mathbf{r}}$ defined by the usual relation

$$
\hat{\mathbf{r}} \psi(\mathbf{r})=\mathbf{r} \psi(\mathbf{r}),
$$

is just $\mathbf{r}$.

The transition from the exact Dirac equation in an external field to its approximate form containing only the first-order correction in $c^{-2}$, is performed just by means of the FW transformation. Thus, in the resulting $c^{-2}$ Hamiltonian the coordinate of a spinning electron is the same $\mathbf{r}$ as in the completely nonrelativistic case 3 .

One more limiting case, which is of a special interest to us, is a classical spinning particle. Such a particle is in fact a well-localized wave packet constructed from positive-energy states, i.e., it is naturally described in the FW representation. Therefore, it is just $\mathbf{r}$ which it is natural to consider as the coordinate of a relativistic spinning particle.

A certain subtlety here is that in the Dirac representation the operator $\hat{\mathbf{r}}$ is nondiagonal. However, the operator equations of motion certainly have the same form both in the Dirac and Foldy-Wouthuysen representations. Correspondingly, the semiclassical approximation to both is the same. In particular, the time derivatives in the left-hand-side of classical equations of motion are taken of the same coordinate $\mathbf{r}$, which serves as an argument of the fields in the right-hand-side of these equations.

As to the covariant operator $\hat{\mathbf{x}}$, it has the simplest form in the Dirac representation:

$$
\hat{\mathbf{x}}_{D}=\sqrt{\frac{\varepsilon}{m}} \beta \mathbf{r}_{D} \sqrt{\frac{\varepsilon}{m}}
$$

where $\hat{\mathbf{r}}_{D}$ is the operator acting on the wave function in the Dirac representation according to the rule (39). The covariance of the matrix element $\psi^{\dagger} \hat{\mathbf{x}} \psi$ is obvious: the matrix $\beta$ transforms $\psi^{\dagger}$ into $\bar{\psi}$, and the factors $\sqrt{\varepsilon / m}$ are needed for the covariant normalization of the wave functions.

Let us rewrite the operator $\hat{\mathbf{x}}$ in the FW representation. The matrix $U$ of the FW transformation is

$$
U=\frac{m+\varepsilon-\beta \boldsymbol{\alpha} \mathbf{p}}{\sqrt{2 \varepsilon(m+\varepsilon)}} .
$$

The calculation, which is conveniently performed in the momentum representation where $\mathbf{r}_{D}=i \boldsymbol{\nabla}_{\mathbf{p}}$, results in the following expression:

$$
\hat{\mathbf{x}}_{F W}=U^{\dagger} \hat{\mathbf{x}}_{D} U=\beta\left(\mathbf{r}+\frac{1}{m(m+\varepsilon)} \mathbf{s} \times \mathbf{p}\right)-\frac{1}{2 m}[(\boldsymbol{\alpha} \mathbf{p}) \hat{\mathbf{r}}+\hat{\mathbf{r}}(\boldsymbol{\alpha} \mathbf{p})] .
$$

\footnotetext{
${ }^{3}$ This is why nobody makes substitution (37) in the Coulomb potential when treating the spin-orbit interaction in the hydrogen atom.
} 
Here

$$
\mathbf{s}=\frac{1}{2}\left(\begin{array}{cc}
\boldsymbol{\sigma} & 0 \\
0 & \boldsymbol{\sigma}
\end{array}\right)
$$

is the relativistic operator of spin. Let us note that different components of the relativistic coordinate operator (42) do not commute. If we confine to the space of the positive-energy states, then we can put in (42) $\beta=1$ and drop the terms with $\boldsymbol{\alpha}$. In this way we arrive at expression (38).

\subsection{Back to Gravitational Interaction}

Thus, there is however a serious problem with the covariant formulation of the equations of motion of a spinning relativistic particle. This equation, for instance, in the gravitational field,

$$
\frac{D}{D \tau}\left(m u_{\mu}-S_{\mu \nu} \frac{D u^{\nu}}{D \tau}\right)=-\frac{1}{2} R_{\mu \nu \rho \sigma} u^{\nu} S^{\rho \sigma},
$$

contains the third time-derivative. As long as the term

$$
-\frac{D}{D \tau}\left(S_{\mu \nu} \frac{D u^{\nu}}{D \tau}\right)
$$

with the third time-derivative, is treated perturbatively, no special problem arises with it by itself. However, the inherent shortcoming of equation (44) (pointed out above for the corresponding electromagnetic equation in the $c^{-2}$ approximation) is that beyond the perturbation theory it evidently has spurious, nonphysical solutions.

But let us confine in (44) to the leading in spin approximation:

$$
\frac{D u_{\mu}}{D \tau}=-\frac{1}{2 m} R_{\mu \nu \rho \sigma} u^{\nu} S^{\rho \sigma}
$$

We note that the right-hand-side of this equation is the only covariant structure possible here (up to a numerical factor). For the nonrelativistic motion in the gravitational field created by a mass $M$, equation (45) reduces to

$$
\ddot{\mathbf{x}}=-\frac{k M}{x^{3}} \mathbf{x}+3 \frac{k M}{m x^{3}}[\mathbf{v} \times \mathbf{s}-(\mathbf{n v}) \mathbf{n} \times \mathbf{s}-2 \mathbf{n}(\mathbf{n}[\mathbf{v} \times \mathbf{s}])], \quad \mathbf{n}=\frac{\mathbf{x}}{x} .
$$

However, the equation of motion that follows from the Lagrangian with potential (26) is somewhat different:

$$
\ddot{\mathbf{r}}=-\frac{k M}{r^{3}} \mathbf{r}+3 \frac{k M}{m r^{3}}\left[\mathbf{v} \times \mathbf{s}-\frac{3}{2}(\mathbf{n v}) \mathbf{n} \times \mathbf{s}-\frac{3}{2} \mathbf{n}(\mathbf{n}[\mathbf{v} \times \mathbf{s}])\right], \quad \mathbf{n}=\frac{\mathbf{r}}{r} .
$$

The reason of the disagreement between (47) and (46) is that they refer to the coordinates $\mathbf{r}$ and $\mathbf{x}$ defined differently (see (37)).

This discrepancy was pointed out long ago in [20] where the classical result (26) was derived from the scattering amplitude for the Dirac particle in the gravitational field. 
Quite typical explanation of the discrepancy was: "The quantum field theory of the spin$1 / 2$ particle from which the classical result was derived does not have any spin supplementary condition $\left(u^{\mu} S_{\mu}=0\right.$ or $\left.u^{\mu} S_{\mu \nu}=0\right)$. This is because field theories deal with point particles and not with extended bodies." In fact, first of all, spin in the Dirac theory certainly satisfies the mentioned constraint (in the sense of expectation values). On the other hand, is the proton in a gravitational field a point particle or an extended body? The deuteron? The uranium nucleus? Obviously, an extended body can be treated as a point particle, as long as we do not go into details of its structure and as long as we do not consider its internal excitations. This point should be emphasized since up to now one may hear utterances similar to the above one on "point particles and extended bodies", even from some well-known theorists.

\section{References}

[1] M. Mathisson, Acta Phys. Polon. 6, 163 (1937).

[2] A. Papapetrou, Proc. Roy. Soc. London A 209, 248 (1951).

[3] W. Pauli, Collected Scientific Papers, ed. by R. Kronig, V.F. Weisskopf (John Wiley and Sons, 1964), v. 2, p. 544.

[4] T.O. Niinikoski, R. Rosmanith, Nucl. Instr. Meth. A 225, 460 (1987).

[5] K. Heinemann, DESY report DESY 96-229;

E-print archive physics/9611001.

[6] K.S. Thorne, J.B. Hartle, Phys. Rev. D 31, 1815 (1985)

[7] L.E. Kidder, C.M. Will, A.G. Wiseman, Phys. Rev. D 47, R4183 (1993)

[8] L. Blanchet, T. Damour, B.R. Iyer, C.M. Will, A.G. Wiseman, Phys. Rev. Lett. 74, 3515 (1995)

[9] H.T. Cho, Class. Quantum Grav. 15, 2465, (1998);

E-print archive gr-qc/9703071

[10] I.B. Khriplovich, A.A. Pomeransky, Phys. Lett. A 216, 7 (1996);

E-print archive gr-qc/9602004.

[11] A.A. Pomeransky, I.B. Khriplovich, Sov. Phys. JETP 86, 839 (1998);

E-print archive gr-qc/9710098.

[12] J. Frenkel, Z. Phys. 37, 243 (1926).

[13] L.H. Thomas, Phil. Mag. 3, 1 (1927).

[14] V. Bargman, L. Michel, V. Telegdi, Phys. Rev. Lett. 2, 435 (1959). 
[15] L.H. Thomas, Nature 117, 514 (1926).

[16] Ya.S. Derbenev, A.M. Kondratenko, Sov. Phys. JETP 37, 968 (1973).

[17] L.D. Landau, unpublished.

[18] A.D. Fokker, Kon. Akad. Weten. Amsterdam. Proc. 23, 729 (1921).

[19] T.A. Apostolatos, Class. Quantum Grav. 13, 799 (1996).

[20] B.M. Barker, R.F. O'Connell, Gen. Rel. Grav. 5, 539 (1974). 